\title{
Research on the Process Layer and Substation Layer Relay Protection in Smart Substation Relay Protection
}

\author{
Kong Haibo, Xie Xiaodong, Wang Lingfei, Sun Peng, Xu Bing \\ Linyi Electric Power Distribution Company of State Grid Shandong Electric Power Company, Linyi, Shandong,
} 276000

\begin{abstract}
The intelligent substation relay protection facility is a fundamental element in construction of modern power system, based on the principles of flexibility, security and accuracy, and it can be divided into process layer and substation layer according to specific equipment. The former is suitable for individual sampling of disposable equipment, can be separated from the base station synchronization sampling, with the help of synchronous digital system and ping-pong algorithm to achieve synchronization of the overall sampling, that is to take the distribution protection components, through the separate transmission medium to realize the information transmission; the latter is for variable power plant components centralized backup protection, taking online real-time tuning technique.
\end{abstract}

Key words: lectric power system; intelligent substation; relay protection

Published Online: 31st Jan 2018

\section{Introduction}

Compared with general substations, intelligent substations have an increasingly wide range of applications and technologies in relay protection emerge endlessly, however, there are relatively less explorations of systematic structures so far. In whether intelligent substations or general substations, the systematic structures are similar. It is proven that this systematic structure can safeguard safety and reliability of substations effectively and overall consistence of various present electric power equipment. Generally, in contrast with normal substations, new intelligent substations use optical cables as a transmission carrier, and optical signals based on coding technologies replace previous electrical signals to ensure real-time effectiveness of sampling and timeliness of information feedback.

Besides, the advantages of general relay protection are inherited and quick trips and auxiliary backup protections at various stages are set up for all the electric power equipment. However, the protection setup for this portion now has yet to be appropriately adjusted and improved by combining specific characteristics of intelligent substations thoroughly.

Accordingly, in this paper, a set of configuration plan for relay protection of intelligent substations is designed. Generally, substation equipment is divided into process layers, which is suitable for individual sampling of one-time devices and can be separated from synchronous sampling of base stations and ensure distributed installation and double-fold configurations, and substation layers, which uniformly set the voltages of various parts of electric power systems for concentrated backup protection of electric power components, are based on self-induction and timely maintenance technologies and have wide-area protection interfaces.

\section{Configuration of relay protection of intelligent substation}

For configuration of relay protection of intelligent substation, the main objectives include process layers and substation layers. The process layer is largely responsible for individual protection of one-time equipment and is divided into two types: First, one-time devices are intelligent devices and play a protective role in the installation of equipment; Second, in case of 
improvement of old equipment, repaired and modified equipment etc. should be installed in the nearby control cabinet uniformly to ensure normal operation and subsequent repair of equipment. GOOSE and sampling values are transmitted and received via Ethernet and timeliness is guaranteed based on IEEE1588. Detailed configuration of relay protection of intelligent substations is shown in the following Fig. 1.

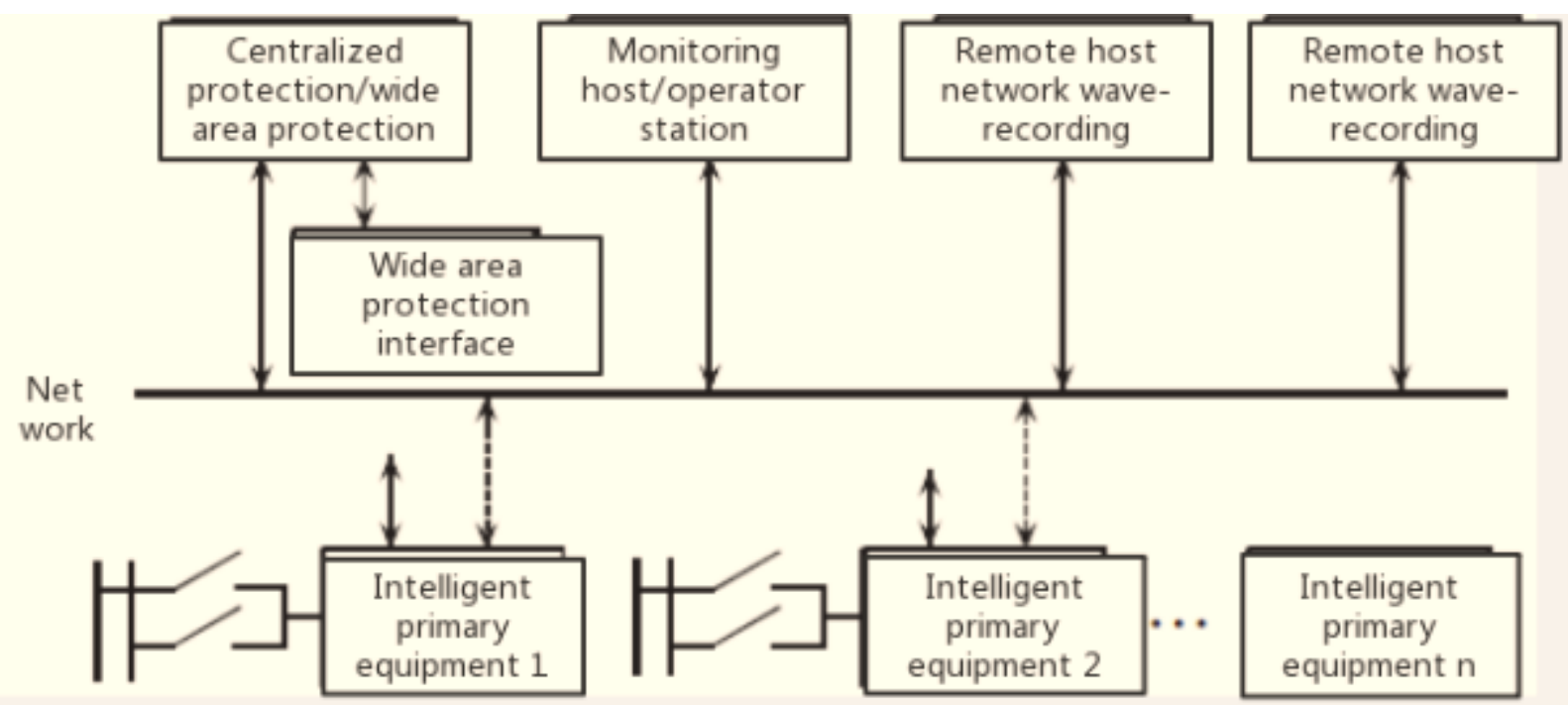

Figure 1. Configuration diagram for relay protection of the intelligent substation

According to the principle of distribute installation of the base station, relay station can be streamlined and the minimal distance between substation equipment and secondary equipment is guaranteed to avoid protection failure resulting from tripping, sampling etc. On top of that, data acquisition and processing of the whole substation rely on consumption of functions like internet monitoring and tests and relay protection reduces this portion of consumption.

\section{Relay protection of the process layer}

Generally, process layer protection, the process layer protection is aimed at line protection for sudden trips, such as circuit pilot and busbar differential and the backup protection of the substation layer are realized through concentrated units and mainly responsible for primary protection. Based on this, devices related to back protection can be simplified to achieve the goal of streamlining system hardware. Besides, fixed values of protection equipment are tuned more stably and will not vary with changes in the operating status of electrical systems basically.

However, due to the independence principle of relay protection, after the concentration of protection equipment for the process layer and the substation layers, if circuits and busbars are protected only via one switch, then their devices should become independent and can be set as independent functional components. The functions of the process layer are elaborated in the following paragraphs:

\subsection{Circuit protection function}

As regards line protection functions in the process layer, primary protection is designed according to the principles of pilot routes and pilot differentials and backup protection equipment is integrated into the protection unit. For single-broken- circuit-type primary wiring, circuit protection is realized with the help of primary protection of fiber-optic communication and test line protection, i.e. pilot protection, with the details seen in Figure 2. 


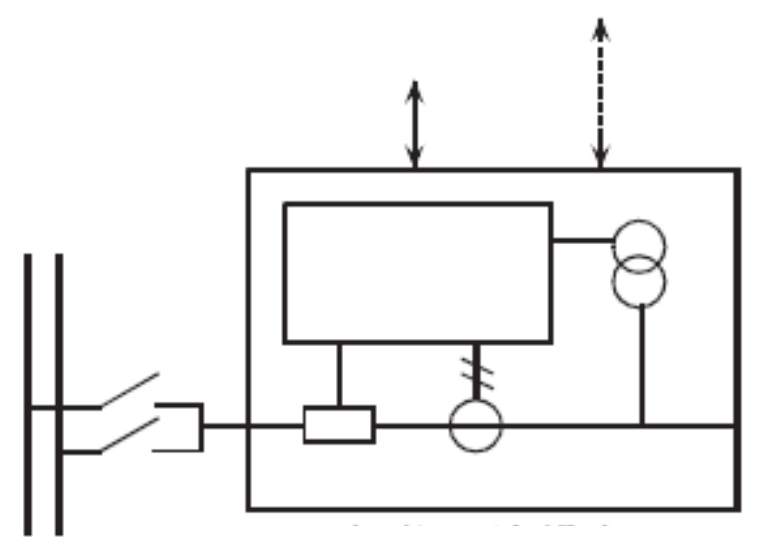

Intelligent primary equipment

Figure 2. Pilot protection illustration of single-broken-circuit-type circuit

In terms of the form of $3 / 2$ line connection, the values of voltage and switching current on the serial line should be collected with details seen in Fig. 3. According to circuit pilot principles, integrated protection equipment does not require acquisition voltage values, however, some special work forms or protection schemes still need collection of values. In this case, voltage values can be collected separately and connected to transmission lines of primary protection data to accomplish the acquisition of it and current values jointly. Due to reliance on same circuits, various switching current values can be acquired separately and data synchronization is realized through transmission of primary protection data. The standard for transmission lines of primary protection data in this mode is relatively high.

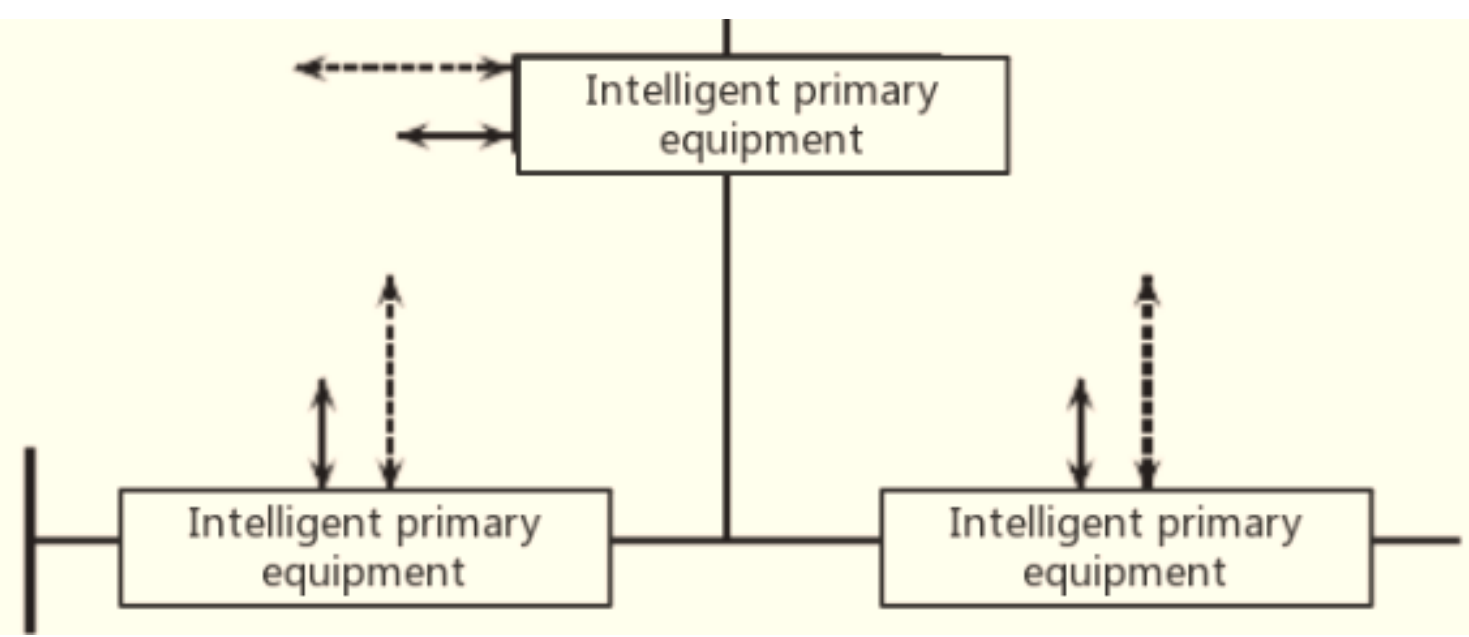

Figure 3. Protection illustration of lines in $3 / 2$ line wiring forms

\subsection{Protection functions of transformer equipment}

For transformer equipment protection, distributed protection units should be used based on differential protection principles. Likewise, backup protection sill adopts integrated units. For non-electricity protection, separate installation should apply with electrical cable connection for circuit breaker tripping, and tripping commands are connected to sampling and GOOSE computers via optical cables. The protection illustration of transformer equipment with $2 / 3$ connection is shown in Figure 4. 

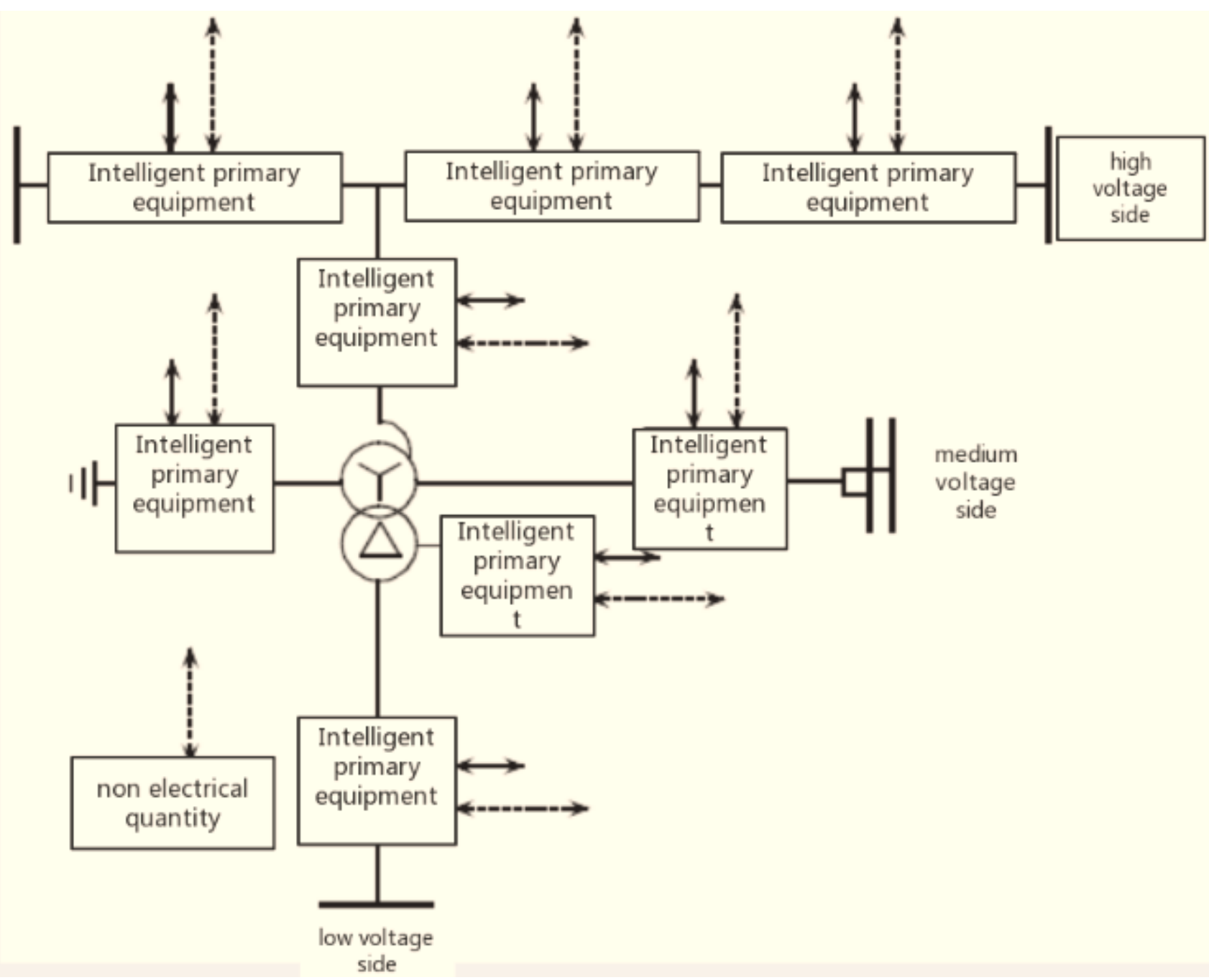

Figure 4. Protection illustration of transformer equipment with $2 / 3$ connection

\subsection{Protection functions of reactors}

In general, reactor protection and transformer equipment protection are roughly the same. The illustration of reactor protection is shown in the following Figure 5.

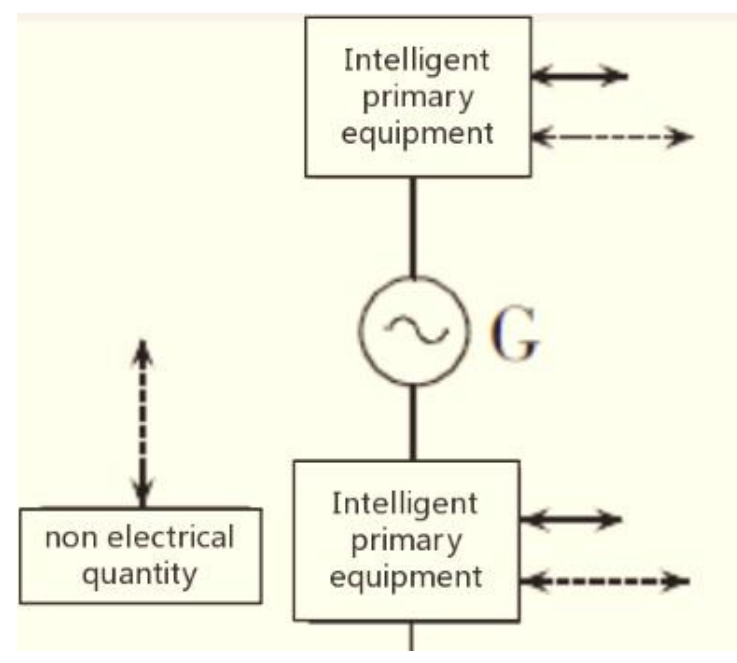

Figure 5. Illustration of reactor protection

\subsection{Busbar protection functions}

In terms of relay busbar protection, protection units are also installed on a distribute basis and various portions realize the busbar protection goal separately. Once a failure occurs, it only leads to tripping of failed circuits. Moreover, Failure-Safe uses integrated protection forms. The illustration of busbar protection is shown the Figure 6 below. 


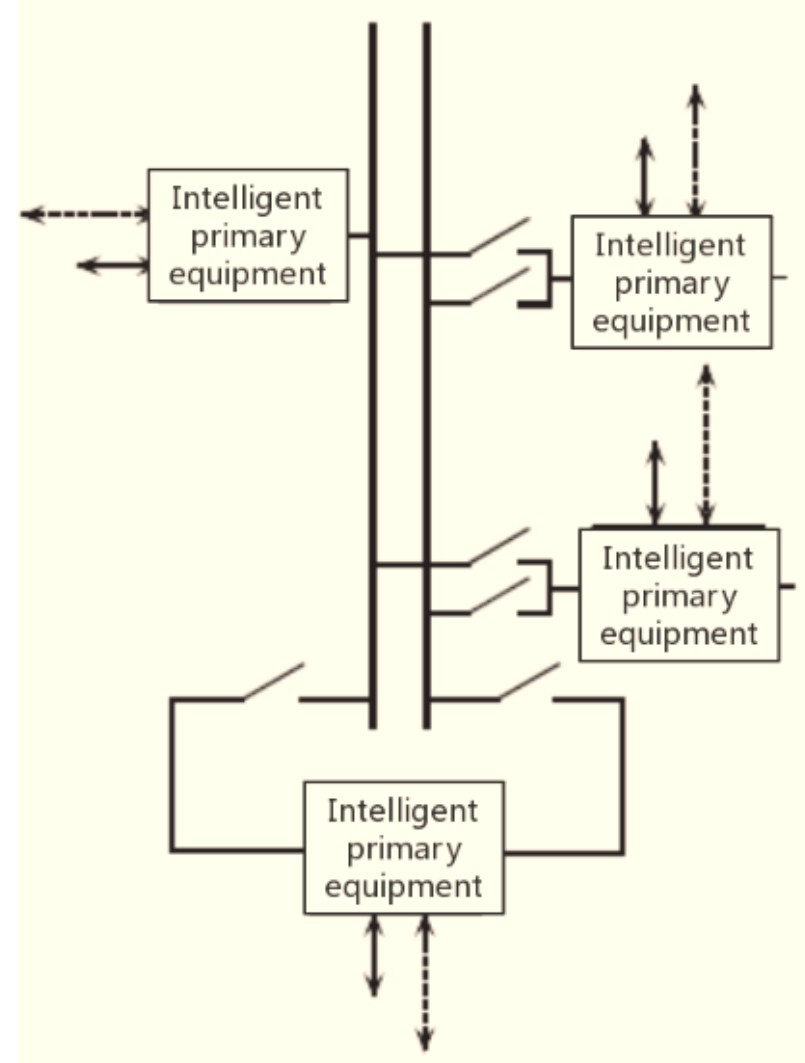

Figure 6. Illustration of busbar protection

\subsection{Adoption of synchronous protection}

Protection units for various functions of the substation should be considered as multi-terminal line protection and synchronize acquisition of sample values for various devices is realized through synchronous protection. The Ping-Pong principle should be utilized effectively based on the rule of unit simplification. It requires the protection devices at the first end and final end of the circuit to collect sample values separately in the same frequency and remain consistent in the time for receipt and transmission of data. The synchronization technology based on the Ping-Pong principle currently and generally used in our country is divided into sample value modification technology and sampling time adjustment technology.

For sample value modification technology, called synchronous vector technology, its basic synchronization principle is shown in the following Fig. 7. $\mathrm{M}$ and $\mathrm{N}$ is two opposite sides, information transmission cycle is $T, t_{P m 1} 、 t_{P m 2} 、 t_{P n 1} 、 t_{P n 2}$ are the sample moment for values at the opposite sides, are the difference between the moment of receipt of opposite-side information by various sides and this side's previous information transmission moment, and $t_{\text {delay }}$ is the duration of transmission between the two sides. The previous data that the opposite side sends to this side $\mathrm{M}_{1}$ and the opposite side previous $\Delta \mathrm{t}_{1}$, this side's latest data $\mathrm{M}_{2}$, the difference between the moment of receipt of information from the opposite side and the moment of this side's previous transmission of information $\Delta \mathrm{t}_{2}$, If the delays in the information transmission of both sides are identical, it is possible to obtain the value ta $=\left(\Delta \mathrm{t}_{2}+\Delta \mathrm{t}_{1}\right) / 2$, ta is the time for this side relative to Pn2 information of the opposite, however, the information collection moment of this side is $\mathrm{t}_{\mathrm{Pm} 2}$, the difference between the times for both sides $\Delta \mathrm{ts}=[$ ta- $(\mathrm{tPm} 2-\mathrm{tPm} 1)]$, the relative angle is $\Delta \theta$, the amount of delayed time for transmission lines $t_{\text {delay }}=[\Delta t 2-\Delta t 1] / 2$. This synchronization technology ensures separated sampling at the first end and final end of lines, and analyzes and adjust the delayed time for information receipt and transmission of transmission lines $t_{\text {delay. }}$. As a result, if the transmission line is intervened, normally it will not affect synchronous sampling.

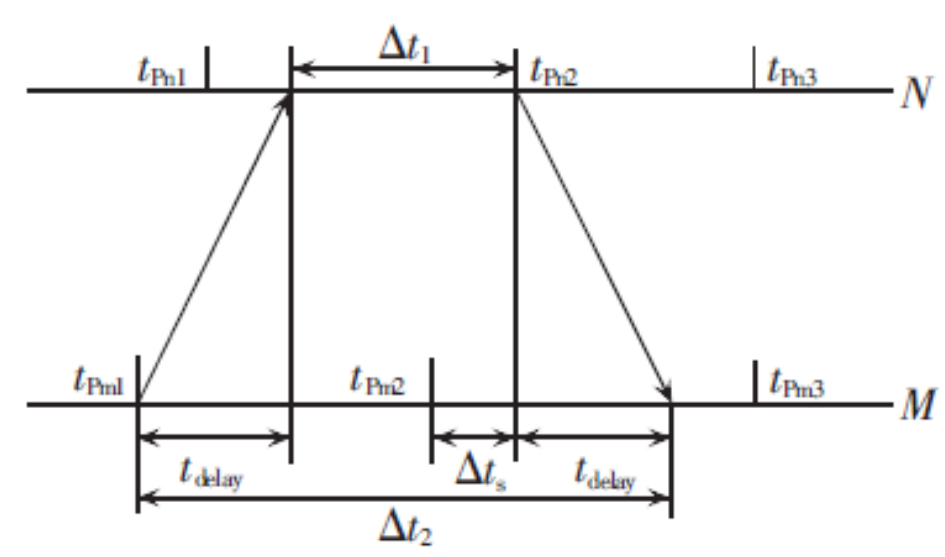

Figure 7. Application illustration of sample value modification technology

Furthermore, sampling time adjustment technology guarantees sampling singleness of the substation and carries out timely control and adjustment through combination of sample moments within the station to ensue high accuracy of synchronous sampling at both sides.

Based on the simplification principle of protection units, the protection unit that realizes synchronization functions is divided into:

(1)Disruptions of information receipt and transmission in the fiber-optic transmission lines is separated. In the previous protection units, both parts are subject to the same disruption control. For electronic sensing devices, 
information sampling is through the sampling portion and information transmission is through the transmission portion. Resulting delays can be obtained by means of measurement.

(2)Information transmission moments at both sides are controlled and adjusted. With the sampling time adjustment technology based on Ping-Pong principles, the information synchronization for both sides can be guaranteed and the consistence of information transmission moments of protection units at both sides is realized.

(3) For the adjustment of sampling delays and transmission delays, it can be analyzed with the help of the following Fig. 8 to ensure synchronization of information receipt moment for both sides.

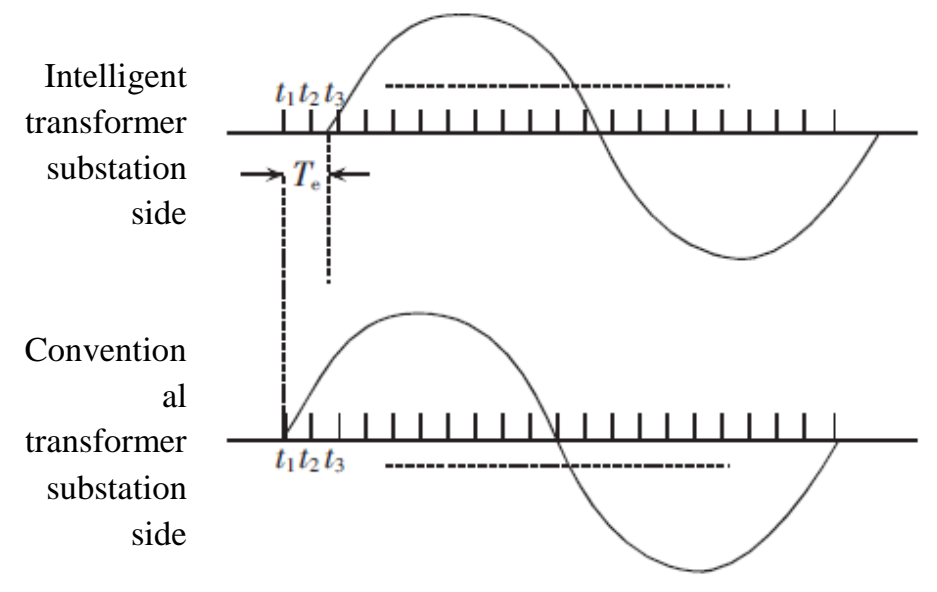

Figure 8. Illustration of delay adjustment

Distributed protection data transmission

Currently, distributed protection data transmission uses SDH system and data transmission protection units are set as transmission circulation, using 3/2 line connection mode on one hand and single-broken-circuit-type connection mode on the other hand to achieve protection goals, with details shown in Figure 9. 


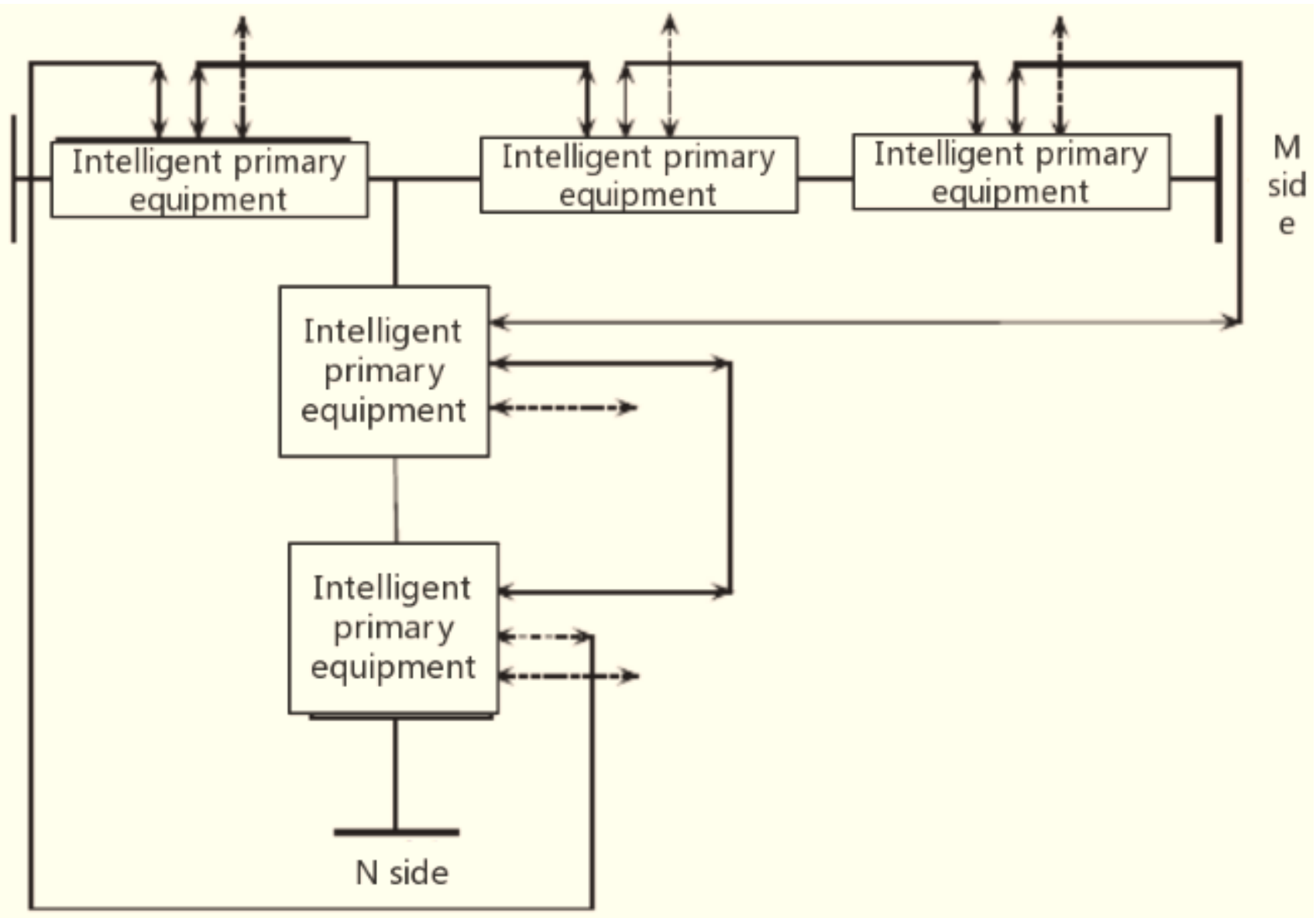

Figure 9. Illustration of transmission circulation of synchronous data

According to the illustration of transmission circulation of synchronous data, two design production programs are particularly available:

First, data transmission synchronization in the substation is for the purpose of synchronization functions only and has low requirements for transmission speed of network information. Sample values collected are communicated via Ethernet. Therefore, various electric power devices can obtain required information from the network.

Second, the whole relay protection unit not only needs to guarantees data synchronization, but also needs to realize data receipt and transmission. It is required to realize transmission of interval information in $1 \mathrm{~ms}$, given the maximum moment difference of the busbar. In addition to featuring synchronous redundancy design, this program sets information transmission functions.

Synchronous system can set some protection unit as primary protection, while the secondary protection helps it realize transmission disruption synchronization.

\section{Relay protection of the substation layer}

The substation layer is mainly aimed at concentrated backup protection and sets various portions of voltages of electrical systems uniformly. Based on self-induction and timely maintenance technologies and with wide-area protection interfaces, it realizes dualization of protection configuration.

Generally, in addition to having a role in the protection of proximal backup and switches for the main substation, this system offer distal backup protection for the auxiliary substation. Hence, the protection content of any substation includes: (1) proximal backup protection is mainly aimed at the busbar and other lines of this substation; (2) the objective of distal backup protection is busbars at the connection terminal and other lines at the connection terminal.

In fact, the backup protection system is mainly responsible for collection and analysis of basic data of the main substation lines and basic status of circuit breaking devices etc., and accommodates short-circuit cases, basic data of lines etc., of the surrounding auxiliary substations. Defective components in the electrical system are judged and effective avoidance programs are fed back through combination of information and data detected. Besides, corresponding 
algorithms are determined in advance according to the working route of the circuit through offline fixed value tuning calculation approaches. Substation protection configuration identifies various working routes according to specific parameters of electrical systems, protection configurations are also adjusted to the set scope of fixed values and thus the goal of protecting action performances is achieved. In addition, this system should include low-voltage or low-frequency protection, over-load line-breakage and other configurations.

\section{Conclusion}

Compared with general substations, intelligent substations have an increasing wide range of applications and technologies in relay protection emerge endlessly. However, there are relatively less researches on systematic structures so far. Effective protection setup and design are carried out for components like substation lines, transformer devices and busbars which are installed in the intelligent devices on a distributed basis to streamline systematic protection settings and reduce difficulties in inspection and repair of relay protection. Besides, for backup protection, integrated configurations are performed to guarantee mutual support of substation protections.

\section{References}

[1] Zhang Zhirui, Xiao Fan, Jiao Shaolin, Dong Wenxiu, Wang Feng, Zhang Zhe, Yin Xianggen. Analysis of reliability of protection system of network structures in different process layers $[\mathrm{J}]$. Power System Protection and Control. 2013(18)

[2] Huang Minghui, Shao Xiangchao, Zhang Chi, Wang Haizhu, Li Yiquan, Cai Zexiang. Modeling and Simulation of relay protection of an intelligent substation based on OPNET[J].Electric Power Automation Equipment.2013(05)

[3] Wang Chao, Wang Huifang, Zhang Chi, Liu Wei, Li Yiquan, He Benteng. A study on modeling of reliability of digital substation relay protection systems [J]. Power System Protection and Control. 2013(03)

[4] Liu Ning, Zhou You, Cai Ming, Li Jun, Chen Wujia, Xu Guangwei. Study and application of evaluations on operating status of secondary equipment for intelligent substations [J].
AUTOMATION \& INSTRUMENTATION. 2016 (03)

[5] Chen Xiaojie. Comparison of tripping modes of intelligent substation protection units $[\mathrm{J}]$ Electric Power and Electrical Engineering. 2011 (04)

[6] Fan Chen,Ni Yimin, Dou Renhui, Shen Jian, Gao Chunlei, Huang Guofang. Analysis of network Scheme for process layer in smart substation [J].Automation of Electric Power Systems. 2011 (18)

[7] Hou Weihong, Zhang Peichao, Hua Yan, Su Yongchun. Analysis of protection system based on highly reliable automated network and its reliability [J]. Power System Protection and Control. 2011 (18)

[8] Zhu Lin, Chen Jinfu, Duan Xianzhong. Evaluation of improvement, reliability and economy of redundant system structure of digital substation $[\mathrm{J}]$. Transactions of China Electrotechnical Societ. 2009 (10)

[9] Zhang Peichao, Gao Xiang. Analysis of reliability and component importance of full digital protection system [J].Proceedings of the CSEE. 2008 (01)

[10] Huo Chao, Zhang Peichao. Analysis of components importance of full digital protection system considering economy $[\mathrm{J}]$. Automation of Electric Power Systems. 2007 (13)

[11] Liu Ning, Zhou You, Cai Ming, Li Jun, Chen Wujia, $\mathrm{Xu}$ Guangwei. Study and application of evaluations on operating status of secondary equipment for intelligent substations [J]. AUTOMATION \& INSTRUMENTATION. 2016 (03)

[12] Du Shuangyu, Wang Xianpei, Xie Guangben, Cai Tao, Zhang Qilin. Evaluation of reliability of substation automation system based on IEC61850 [J]. Power System Protection and Control. 2012 (05)

[13] Zhu Shenghui, Zhou Fuqiang, Xia Han, Ni Zhenqiang. Exploration on replay protection means of intelligent substation based on spatial dimensions [J]. AUTOMATION \& INSTRUMENTATION. 2016 (02)

[14] Shen Yuan. Study of an N-k risk approach for grid considering multi-mode concealed failures in relay protection system $[\mathrm{J}]$. AUTOMATION \& INSTRUMENTATION. 2015 (11)

[15] ANDERSSON L,BRAND K P,BRUNNER C,et al.Reliability Investigations for SA Communication Architectures Based onIEC61850. Proceedings of IEEE Power Tech 2005. 2005 